\title{
Parameter Reduction Method for Pythagorean Fuzzy Soft Sets
}

\author{
Murat Kirişci $^{1}$ \\ ${ }^{1}$ Department of Mathematical Education, Istanbul University-Cerrahpaşa, Vefa, 34470, Fatih, Istanbul, Turkey
}

\section{Article Info}

Keywords: Parameter reduction, Pythagorean fuzzy soft set. 2010 AMS: 03E72, 68737

Received: 4 September 2019

Accepted: 14 October 2019

Available online: 25 March 2020

\begin{abstract}
The aim of this paper is to give new parameter reduction methods according to Pythagorean fuzzy soft sets. The reason for the definition of these methods is to help decision-makers facilitate their decision-making processes. The algorithm of the first method defined is related to the selection of some parameters. The second method determines the parameters with less deviation than the other parameters. Further, numerical examples related to the new algorithms are examined.
\end{abstract}

\section{Introduction}

Fuzzy Sets (FSs) put forward by Zadeh [1] has influenced deeply all the scientific fields since the publication of his paper. It is seen that this concept, which is very important for real-life situations, had not enough solution to some problems in time. New quests for such problems have been coming up. Atanassov [2] initiated Intuitionistic fuzzy sets (IFSs) for such cases.

The SS theory, which contributed to the solution of uncertainties in non-parametric problems, was initiated by Molodtsov [3]. SS theory is an inherent extension of the FS theory and can, therefore, be easily applied to all branches of science and technology. This theory deals with a concentration of approximate illustration of objects. In the approximate illustration, there exists a set of predicate values and a set of approximate values. This theory is suitable and simply operative in performance due to the nonentity of restrictions on the approximate illustrations.

Yager [4] offered a new FS called Pythagorean fuzzy set(PFS). PFS has fascinated the care of great deal researchers in a little while time. The formulation of the negation for IFSs and PFSs is examined by Yager [5]. In [6], PF subsets and its relationship with IF subsets were debated and some set operations on PF subsets were defined. Peng et al. [7], given the definition of the Pythagorean fuzzy soft set(PFSS), investigated its properties. Kirisci [8] introduce Pythagorean fuzzy parametrized soft set(PFPSS) and examine some characteristics, operations. In addition, the answer of the decision-making(DM) problem with PFPSS and other related notions is presented in [8].

\section{Preliminaries}

Give the sets $\mathscr{U}, P, \rho(\mathscr{U})$ as an initial universe, parameters, the power set of $\mathscr{U}$ respectively, and $S \subset P$. Give the mapping $m: X \rightarrow \rho(\mathscr{U})$. Therefore, $u_{X}$ is said to a soft $\operatorname{set}(\mathrm{SS})$ over $U$ [3].

Choose the set $\mathscr{U}=\left\{x_{i}\right\}_{i=1}^{n}$. Let $\{S(j)\}_{j=1}^{k}$ be a set of parameters.

Let $\left\{\cup_{j=1}^{k} S(j)\right\} \subseteq P$ and every parameter set $S(k)$ indicate the $k$ th class of parameters and the elements of $S(k)$ represents a exclusive attribute set.

The set

$$
A=\left\{\left\langle x, u_{A}(x), v_{A}(x)\right\rangle: x \in \mathscr{U}\right\}
$$


is said to be an intuitionistic fuzzy $\operatorname{set}(\mathrm{IFS}) A$ on $\mathscr{U}$, [2] where, $u_{A}, v_{A}: \mathscr{U} \rightarrow[0,1]$ such that $0 \leq u_{A}(x)+v_{A}(x) \leq 1$ for any $x \in \mathscr{U}$. The degree of indeterminacy $w_{A}=1-u_{A}(x)-v_{A}(x)$.

An Pythagorean fuzzy set(PFS) $\varphi$ over $\mathscr{U}$ is given by

$$
\varphi=\left\{\left\langle x, u_{\varphi}(x), v_{\varphi}(x)\right\rangle: x \in \mathscr{U}\right\},
$$

where $u_{\varphi}, v_{\varphi}: \mathscr{U} \rightarrow[0,1]$ denote the degree of membership and the degree of non-membership of $x \in \mathscr{U}$ to $\varphi$, respectively, such that $0 \leq\left(u_{\varphi}(x)\right)^{2}+\left(v_{\varphi}(x)\right)^{2} \leq 1[4,5] . \mathscr{I}_{\varphi}=\sqrt{1-\left(u_{\varphi}(x)^{2}-\left(v_{\varphi}(x)\right)^{2}\right.}$ represent the degree of indeterminacy.

Then, $\varphi(S)$ is called Pythagorean Fuzzy Soft Set(PFSS) on $\mathscr{U}$, if $\varphi(S)$ is mapping given by $\varphi(S): P \rightarrow \rho(\mathscr{U})$ [7].

Remark 2.1. It is easy to check that PFSSs generalize both IFSs and SSs. That is, all IF degrees are part of the PF degrees. In actual DM problems, the PFSS characterizes a larger membership space than the IFSS. That is, the PFSS a higher capability than the IFSS to model uncertainty in real DM problems.

Let Pythagorean fuzzy numbers (PFNs) are denoted by $E=\left(u_{S}, v_{S}\right)$ [9]. Choose three PFNs $\theta=E(u, v), \theta_{1}=\left\langle u_{1}, v_{1}\right\rangle, \theta_{2}=\left\langle u_{2}, v_{2}\right\rangle$. We can give some basic operations as follows [4]:

- $\bar{\theta}=\langle u, v\rangle$;

- $\theta_{1} \vee \theta_{2}=\left\langle\max \left\{u_{1}, u_{2}\right\}, \min \left\{v_{1}, v_{2}\right\}\right\rangle$;

- $\theta_{1} \wedge \theta_{2}=\left\langle\min \left\{u_{1}, u_{2}\right\}, \max \left\{v_{1}, v_{2}\right\}\right\rangle$;

- $\theta_{1} \oplus \theta_{2}=\left\langle\sqrt{u_{1}^{2}+u_{2} 2-u_{1}^{2} u_{2}^{2}}, v_{1} v_{2}\right\rangle$

- $\theta_{1} \otimes \theta_{2}=\left\langle u_{1} u_{2}, \sqrt{v_{1}^{2}+v_{2}^{2}-v_{1}^{2} v_{2}^{2}}\right\rangle$;

- $\alpha \cdot \theta=\left\langle\sqrt{1-\left(1-u^{2}\right)^{\alpha}}, v^{\alpha}\right\rangle$;

- $\theta^{\alpha}=\left\langle u^{\alpha}, \sqrt{1-\left(1-v^{2}\right)^{\alpha}},\right\rangle$;

for $\alpha>0$.

Maji et al. [10] firstly gave IFSS. In [11], Q-IFSS defined and basic properties are investigated. Broumi et al. [12] are given new definitions for IFSS such as concentration, dilatation and normalization. In [13], first Zadeh's containment, IF conjunction, IF disjunction of two IFSSs are defined and some basic properties are examined. In [14], three new operations based on Second Zadeh's containment, conjunction and disjunction operations have been defined and studied. Maji [15] has been extended IFSS with new operations. In [16], a new approach to IFSS was presented with rough set for DM problems. In this study, we adopt the PFSS from idea of Ghosh and Das [17]. Kirisci [18] compared IFPSS and Riesz summability methods using medical real dataset.

\section{Comparison of IFSs and PFSs}

IFS, offered by Atanassov [2] is an extension of FS Theory [1]. IFS is characterized by a membership degree and a non-membership degree and therefore can indicate the fuzzy character of data in more detail comprehensively. The prominent characteristic of IFS is that it assigns to each element a membership degree and a non-membership degree with their sum equal to or less than 1. However, in some practical DM process, the sum of the membership degree and the non-membership degree to which an alternative satisfying a criterion provided by a decision maker may be bigger than 1, but their square sum is equal to or less than 1 .

Therefore, Yager [4] proposed PFS characterized by a membership degree and a non-membership degree, which satisfies the condition that the square sum of its membership degree and non-membership degree is less than or equal to 1. Yager [19] gave an example to state this situation: a decision maker gives his support for membership of an alternative is $\frac{\sqrt{3}}{2}$ and his against membership is $\frac{1}{2}$. Owing to the sum of two values is bigger than 1, they are not available for IFS, but they are available for PFS since $\left(\frac{\sqrt{3}}{2}\right)^{2}+\left(\frac{1}{2}\right)^{2} \leq 1$. Obviously, PFS is more capable than IFS to model the vagueness in the practical multicriteria decision-making problems.

The main difference between PFNs and IFNs is their corresponding constraint conditions, which can be easily shown in Figure 3.1. Here, we observe that intuitionistic membership grades are all points under the line $x+y \leq 1$ and the Pythagorean membership grades are all points with $x^{2}+y^{2} \leq 1$.

One important implication of this is that it allows the use of the PFSs in situations in which we cannot use IFSs. An example of this would be a case in which a user indicates that their support for membership of $x$ is $\frac{\sqrt{3}}{2}$ and their support against membership is $\frac{1}{2}$. As we noted these values are not allowable for intuitionistic membership grades but allowable as Pythagorean membership grades. Thus in this case, rather then requiring the user to change their information to satisfy the constraints of the IFS we can use a PFS.

\section{Parameter Reduction Method}

Take a complete lattice $(\mathscr{L}, \leq \mathscr{L})$, such that $\mathscr{L}=\left\{(x, y): x, y \in[0,1], x^{2}+y^{2}<1\right\}$. The corresponding partial order $\leq \mathscr{L}$ id defined by $(x, y) \leq \mathscr{L}(a, b) \quad x \leq a, \quad y \geq b, \forall(x, y),(a, b) \in \mathscr{L}$. Any ordered pair $(x, y) \in \mathscr{L}$ is said to be Pythagorean fuzzy value(PFV) or Pyhtagorean 


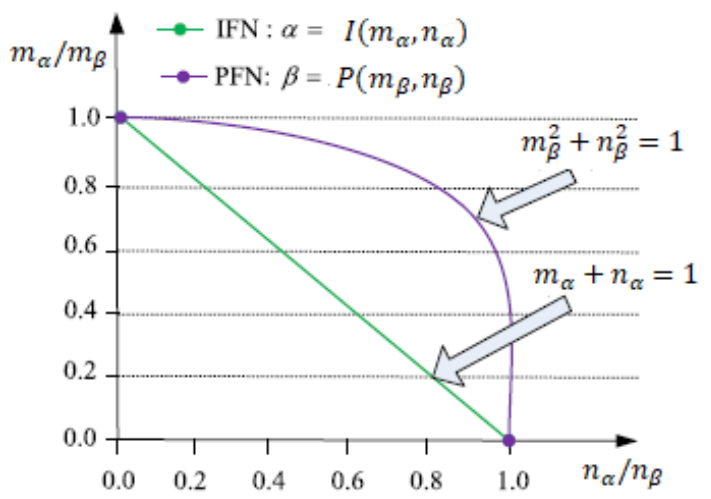

Figure 3.1: The PFNs and the IFNs

fuzzy number(PFN) [20].

Let $E=\left(u_{E}, v_{E}\right)$ be a Pythagorean fuzzy number (PFN). The mapping $\mathscr{S} \mathscr{F}_{E}: L \rightarrow[-1,1]$ is called score function, if $\mathscr{S} \mathscr{F}_{E}=u_{E}^{2}-v_{E}^{2}$ for all $E \in L$ [8], [9].

For any two PFNs $E, F$

$$
\begin{aligned}
E & \prec F, \text { if } \mathscr{S} \mathscr{F}(E)<\mathscr{S} \mathscr{F}(F), \\
E & \succ F, \text { if } \mathscr{S} \mathscr{F}(E)>\mathscr{S} \mathscr{F}(F), \\
E & \sim F, \text { if } \mathscr{S} \mathscr{F}(E)=\mathscr{S} \mathscr{F}(F) .
\end{aligned}
$$

\section{Algorithm 1:}

For each $i, j$, score values(SVs) of each of the entries of the PFSS $\varphi(S)$ denoted by $\phi=\mathscr{S} \mathscr{F}_{E}$. Define the aggregated score as $\psi=\overline{\mathscr{S} \mathscr{F}_{E}}\left(x_{i}\right)=\sum_{j=1}^{m} \mathscr{S} \mathscr{F}_{E}\left(x_{j}\right)$. Consider $\lambda=\left\{x_{i}^{*}\right\}_{i=1}^{r} \subset P$ and $\mu=\left\{x_{i}^{* *}\right\}_{i=1}^{r} \subset P$.
i. Calculate $\phi$
ii. Compute $\psi$.
iii. Select the sets $\lambda, \mu \subset P$, where $\lambda, \mu \neq \emptyset, \lambda \cap \mu=\emptyset, \lambda \cup \mu \neq E$.
iv. Compute $\psi_{\lambda \subset P}, \psi_{\mu \subset P}\left(\forall x_{i} \in \mathscr{U}\right)$.
v. Choose the reduction parameter set of the PFSS $\phi$ as $P-(\lambda \cup \mu)$, if $\psi_{\lambda \subset P}-\psi_{\mu \subset P}=0$.

Example 4.1. Take the set of blouses $U=\left\{y_{1}, y_{2}, y_{3}, y_{4}, y_{5}, y_{6}\right\}$. Parameters can be defined as $p_{1}:$ bright, $p_{2}:$ cheap, $p_{3}:$ costly, $p_{4}:$ very costly, $p_{5}$ :colourful, $p_{6}:$ cotton, $p_{7}:$ polystyrene, $p_{8}:$ long sleeve. The tabular representation of PFFS $\varphi$ is given Table 1 .

Table 2 shows score of each entry and object for the PFSS $\varphi$ (for $i=1,2, \cdots, 6)$.

The order of the blouses is found as $y_{5}, y_{6}, y_{4}, y_{1}, y_{2}, y_{3}$. Now we choose the sets $\lambda, \mu$ such as $\lambda, \mu \subset P$ and $\lambda \cup \mu \neq P$. Consider the set $\lambda=\left\{p_{1}, p_{4}\right\}$ and $\mu=\left\{p_{5}, p_{7}\right\}$. It can be easily seen that reduced parameter set $P^{*}=\left\{p_{2}, p_{3}, p_{6}, p_{8}\right\}$ is obtained, when $\psi\left(y_{i}\right)$ of each object $y_{i}$ are computed. The table which include the parameter set $P^{*}$ is given Table 3. The object ordering in Table 2 and Table 3 appears to be the same.

\begin{tabular}{l|llllllll}
\hline & $p_{1}$ & $p_{2}$ & $p_{3}$ & $p_{4}$ & $p_{5}$ & $p_{6}$ & $p_{7}$ & $p_{8}$ \\
\hline$y_{1}$ & $(0.5,0.3)$ & $(0.4,0.3)$ & $(0.5,0.4)$ & $(0.1,0.6)$ & $(0.3,0.5)$ & $(0.4,0.2)$ & $(0.6,0.1)$ & $(0.1,0.5)$ \\
$y_{2}$ & $(0.2,0.5)$ & $(0.4,0.1)$ & $(0.5,0.2)$ & $(0.1,0.2)$ & $(0.5,0.2)$ & $(0.2,0.3)$ & $(0.2,0.1)$ & $(0.4,0.6)$ \\
$y_{3}$ & $(0.4,0.6)$ & $(0.2,0.5)$ & $(0.4,0.3)$ & $(0.5,0.3)$ & $(0.6,0.4)$ & $(0.3,0.4)$ & $(0.3,0.5)$ & $(0.5,0.4)$ \\
$y_{4}$ & $(0.5,0.4)$ & $(0.3,0.5)$ & $(0.3,0.6)$ & $(0.6,0.2)$ & $(0.2,0.6)$ & $(0.6,0.2)$ & $(0.4,0.5)$ & $(0.4,0.3)$ \\
$y_{5}$ & $(0.3,0.6)$ & $(0.4,0.2)$ & $(0.6,0.3)$ & $(0.6,0.3)$ & $(0.5,0.3)$ & $(0.7,0.1)$ & $(0.3,0.5)$ & $(0.5,0.5)$ \\
$y_{6}$ & $(0.2,0.5)$ & $(0.6,0.2)$ & $(0.6,0.1)$ & $(0.5,0.2)$ & $(0.1,0.7)$ & $(0.3,0.5)$ & $(0.7,0.1)$ & $(0.4,0.5)$ \\
\hline
\end{tabular}




\begin{tabular}{l|lllllllll}
\hline & $p_{1}$ & $p_{2}$ & $p_{3}$ & $p_{4}$ & $p_{5}$ & $p_{6}$ & $p_{7}$ & $p_{8}$ & $\psi$ \\
\hline$y_{1}$ & 0.16 & 0.07 & 0.09 & -0.35 & -0.16 & 0.12 & 0.35 & -0.24 & 0.04 \\
$y_{2}$ & -0.21 & 0.15 & 0.21 & -0.03 & 0.21 & -0.05 & 0.03 & -0.2 & 0.11 \\
$y_{3}$ & -0.2 & -0.21 & 0.07 & 0.16 & 0.2 & -0.07 & -0.16 & 0.09 & -0.12 \\
$y_{4}$ & 0.09 & -0.16 & -0.27 & 0.32 & -0.32 & 0.32 & -0.09 & 0.07 & -0.04 \\
$y_{5}$ & -0.27 & 0.12 & 0.27 & 0.27 & 0.16 & 0.48 & -0.16 & 0 & 0.87 \\
$y_{6}$ & -0.21 & 0.32 & 0.35 & 0.21 & -0.48 & -0.16 & 0.48 & -0.09 & 0.42 \\
\hline
\end{tabular}

Table 2: Score of each object and entry for the PFSS $\varphi$

\begin{tabular}{l|lllll}
\hline & $p_{2}$ & $p_{3}$ & $p_{6}$ & $p_{8}$ & $\psi$ \\
\hline$y_{1}$ & 0.07 & 0.09 & 0.12 & -0.24 & 0.04 \\
$y_{2}$ & 0.15 & 0.21 & -0.05 & -0.2 & 0.11 \\
$y_{3}$ & -0.21 & 0.07 & -0.07 & 0.09 & -0.12 \\
$y_{4}$ & -0.16 & -0.27 & 0.32 & 0.07 & -0.04 \\
$y_{5}$ & 0.12 & 0.27 & 0.48 & 0 & 0.87 \\
$y_{6}$ & 0.32 & 0.35 & -0.16 & -0.09 & 0.42 \\
\hline
\end{tabular}

Table 3: Reduced parameter set

\begin{tabular}{l|lllll}
\hline & $p_{1}$ & $p_{2}$ & $p_{3}$ & $p_{4}$ & $p_{5}$ \\
\hline$y_{1}$ & $(0.65,0.42)$ & $(0.7,0.4)$ & $(0.55,0.12)$ & $(0.9,0.15)$ & $(0.85,0.2)$ \\
$y_{2}$ & $(0.45,0.75)$ & $(0.6,0.4)$ & $(0.33,0.88)$ & $(0.5,0.5)$ & $(0.52,0.5)$ \\
$y_{3}$ & $(0.6,0.3)$ & $(0.8,0.05)$ & $(0.7,0.5)$ & $(0.6,0.3)$ & $(0.7,0.45)$ \\
$y_{4}$ & $(0,35,0.65)$ & $(0.4,0.2)$ & $(0.25,0.75)$ & $(0.4,0.5)$ & $(0.55,0.45)$ \\
\hline
\end{tabular}

Table 4: Tabular representation of PFSS $\varphi$

\begin{tabular}{l|llllll}
\hline & $p_{1}$ & $p_{2}$ & $p_{3}$ & $p_{4}$ & $p_{5}$ & $\psi$ \\
\hline$y_{1}$ & 0.2461 & 0.33 & 0.2881 & 0.7875 & 0.6825 & 2.3342 \\
$y_{2}$ & -0.36 & 0.2 & -0.6655 & 0 & 0.0204 & -0.8051 \\
$y_{3}$ & 0.27 & 0.6375 & 0.24 & 0.27 & 0.2875 & 1.705 \\
$y_{4}$ & -0.3 & 0.12 & -0.5 & -0.09 & 0.1 & -0.67 \\
\hline
\end{tabular}

Table 5: Score values for the PFSS $\varphi$

\begin{tabular}{l|lllll}
\hline & $p_{1}$ & $p_{3}$ & $p_{4}$ & $p_{5}$ & $\psi$ \\
\hline$y_{1}$ & 0.2461 & 0.2881 & 0.7875 & 0.6825 & 2.0042 \\
$y_{2}$ & -0.36 & -0.6655 & 0 & 0.0204 & -1.0051 \\
$y_{3}$ & 0.27 & 0.24 & 0.27 & 0.2875 & 1.0675 \\
$y_{4}$ & -0.3 & -0.5 & -0.09 & 0.1 & -0.79 \\
\hline
\end{tabular}

Table 6: The set of reduced parameter set and score values

\section{Algorithm 2:}

For $i \neq j$, the maximum score deviation denoted by $\sigma=\min \left\{\left|\overline{\mathscr{S} \mathscr{F}_{N}}\left(a_{i}\right)-\overline{\mathscr{S} \mathscr{F}_{N}}\left(a_{j}\right)\right|\right\}$, for $i, j=1,2, \cdots n$.

i. Calculate $\phi$

ii. Compute $\psi$.

iii. Calculate $\sigma$ with $P=\left\{p_{1}, p_{2}, \cdots, p_{m}\right\}$.

iv. Select the parameter $p_{k} \in P$ for the following situations for $i, j=1,2, \cdots, m$

a) For $j \neq k, \mathscr{S} \mathscr{F}_{N}\left(a_{i}, p_{k}\right) \leq \mathscr{S} \mathscr{F}_{N}\left(a_{i}, p_{j}\right)(\forall i)$.

b) $\forall i$ and for $j \neq k, \max \left|\mathscr{S} \mathscr{F}_{N}\left(a_{i}, p_{k}\right)-\mathscr{S} \mathscr{F}_{N}\left(a_{i}, p_{j}\right)\right|<\sigma$.

v. Take the maximal number of parameter set $A$ which fulfill the preceding step.

vi. Calculate the set $P-A$. This set is a reduced version of the parameters of PFSS. 
Example 4.2. Consider the information as given in Table 4. Firstly, compute SVs of $y_{i}, \quad(i=1,2,3,4)$. Obtained SVs are corresponding to the parameters $P$, which is defined as $\psi=\overline{\mathscr{S} \mathscr{F}_{N}}\left(a_{i}\right)=\sum_{j=1}^{m} \mathscr{S}_{\mathscr{F}}\left(a_{i}, p_{j}\right)$.Therefore the objects are ordered with SVs and accuracy values. The order is shown as $\left\{h_{1}, h_{3}, h_{4}, h_{2}\right\}$.

The maximum deviation is computed as $\sigma=\min \{3.1393,0.6292,3.0042,-2.5101,-0.1351,2.375\}=-2.5101$. We consider the parameter $p_{2} \in P$, where $\mathscr{S} \mathscr{F}_{N}\left(a_{i}, p_{k}\right) \leq \mathscr{S} \mathscr{F}_{N}\left(a_{i}, p_{j}\right)$ for all $i, j$ and $\max \left|\mathscr{S} \mathscr{F}_{N}\left(a_{i}, p_{k}\right)-\mathscr{S} \mathscr{F}_{N}\left(a_{i}, p_{j}\right)\right|<\sigma$ for all $i, j$ (Table 5). Thus the reduction of the parameter $p_{2}$ will have no effect in the ordering of objects, which is shown in Table 6. So, we get the reduced set of parameters as $P-\left\{p_{2}\right\}$.

\section{Conclusion}

Parameter reduction is primarily a method used to avoid redundant parameters. The results obtained with the minimum subset of parameters are the same as the entire set of parameters. Parameter reduction is essentially based on this idea. It is proposed new parameter reduction methods for PFSSs. Examples are given to illustrate the applicability of these methods.

\section{References}

[1] L.A. Zadeh, Fuzzy sets, Inf. Comp., 8, (1965), 338-353.

[2] K. Atanassov, Intuitionistic fuzzy sets, Fuzzy Sets and Systems, 20, (1986), 87-96.

[3] D. Molodtsov, Soft Set Theory-First Results, Comput. Math. Appl., 37, (1999), 19-31

[4] R. R. Yager, Pythagorean fuzzy subsets, In: Proc Joint IFSA World Congress and NAFIPS Annual M eeting, Edmonton, Canada; (2013), 57-61.

[5] R. R. Yager, Pythagorean membership grades in multicriteria decision making, IEEE Trans Fuzzy Syst., 22, (2014), 958-965.

[6] R.R. Yager, Properties and applications of Pyhagorean fuzzy sets, in Imprecision and Uncertainty in Information Representation and Processing, Springer, Berlin, (2016), 119-136.

[7] X. Peng, Y. Yang, J. Song, Y. Jiang, Pythagorean fuzzy soft set and its application, Computer Engineering, 41(7), (2015), $224-229$.

[8] M. Kirişci, New type Pythagorean fuzzy soft set and decision-making application, https://arxiv.org/pdf/1904.04064.pdf.

[9] XL Zhang, ZS Xu, Extension of TOPSIS to multi-criteria decision making with Pythagorean fuzzy sets, Int. J. Intell. Syst., 29, (2014), 1061-1078.

[10] P. K. Maji, R. Biswas, A. R. Roy, Intuitionistic fuzzy soft sets, The journal of fuzzy mathematics, 9, (2001), 677-692.

[11] S. Broumi, Q-Intuitionistic fuzzy soft sets, Journal of New Theory, 5, (2015), 80-91.

[12] S. Broumi, F. Smarandache, M. Dhar, P. Majumdar, New results of intuitionistic fuzzy soft set, I.J. Information Engineering and Electronic Business, 2, (2014), 47-52.

[13] S. Broumi, P. Majumdar, F. Smarandache, New Operations on Intuitionistic Fuzzy Soft Sets Based on First Zadeh's Logical Operators, Journal of New Results in Sciences, 4, (2014), 71-81.

[14] S. Broumi, P. Majumdar, F. Smarandache, New Operations on Intuitionistic Fuzzy Soft Sets based on Second Zadeh's logical Operators, 5, (2015), 80-91.

[15] P.K. Maji, More on Intuitionistic Fuzzy Soft Sets. In: Sakai H., Chakraborty M.K., Hassanien A.E., Ślęzak D., Zhu W. (eds) Rough Sets, Fuzzy Sets, Data Mining and Granular Computing. RSFDGrC 2009. Lecture Notes in Computer Science, vol 5908. Springer, Berlin, Heidelberg, (2009).

[16] Z. Zhang, A rough set approach to intuitionistic fuzzy soft set based decision making, Applied Mathematical Modelling, 36, (2012), 44605-4633.

[17] S. Ghsoh, S. Das, Parameter reduction of intuitionistic fuzzy soft sets and its related algorithms, Proceedings of the 4th International Conference on Frontiers in intelligent computing: Theory and Applications(FICTA) (2015).

[18] M. Kirişci, Comparison the medical decision-making with Intuitionistic fuzzy parameterized fuzzy soft set and Riesz Summability, New Mathematics and Natural Computation, 15(2), (2019), 351-359. doi: 10.1142/S1793005719500194.

[19] Yager RR, Abbasov AM. Pythagorean membership grades, complex numbers, and decision making. Int J Intell Syst 2014;28:436-452.

[20] H. Garg, A New Generalized Pythagorean Fuzzy Information Aggregation Using Einstein Operations and Its Application to Decision Making, Int J Intell Syst, 31, (2016), 886-920. 